\title{
A measure of helical propensity for amino acids in membrane environments
}

Shun-Cheng Li and Charles M. Deber

Nature Structural Biology 1, 368-373 (1994).

The abstract and Table 1 were printed with a number of typographical errors which are corrected in the version below.

The frequent occurrence of $\beta$-sheet promoting residues such as Ile, Val, and Thr in the $\alpha$-helical transmembrane segments of most integral membrane proteins suggests that the helical propensities of these residues are altered in the hydrophobic environment of the lipid bilayer. Systematic studies of model peptides by circular dichroism spectroscopy in various micellar/vesicular media allow the establishment of a ranking order of helical propensity for uncharged amino acids in the membrane environment. In contrast to their conformational preferences in water, the helical proclivity of amino acids in membranes is shown to be governed by their side chain hydrophobicity, and by the hydropathy of the local peptide segments in which the residues reside.

Table 1 Primary sequences and selected properties of host-guest peptides

\begin{tabular}{|c|c|c|c|c|c|c|c|}
\hline Residue & Primary Sequences of $\mathrm{AXA} P \mathrm{Pe}$ & & $-\theta_{222}^{2}$ & & & $P_{\alpha}(X)^{3}$ & $\operatorname{Trp} \Delta \lambda_{\max }{ }^{4}$ \\
\hline$x$ & $\begin{array}{cc}5 & 14 \\
\text { SKSK-AXAAXAWAXA-KSKSKS }\end{array}$ & Aq. & SDS & LPG & DMPG & & \\
\hline I & SKSK-AIAAIAWAIA-KSKSKS & 4,800 & 25,700 & 23,900 & 23,000 & 1.08 & 13 \\
\hline $\mathrm{L}$ & SKSK-ALAALAWALA-KSKSKS & 11,600 & 25,100 & 23,400 & 22,900 & 1.21 & 13 \\
\hline V & SKSK-AVAAVAWAVA-KSKSKS & 1,700 & 25,000 & 23,100 & 22,400 & 1.06 & 13 \\
\hline $\mathrm{M}$ & SKSK-AMAAMAWAMA-KSKSKS & 5,800 & 22,700 & 22,200 & 21,700 & 1.45 & 16 \\
\hline $\mathrm{F}$ & SKSK-AFAAFAWAFA-KSKSKS & 1,000 & 21,500 & 21,000 & 19,400 & 1.13 & 13 \\
\hline$A$ & SKSK-AAAAAAWAAA-KSKSKS & 11,300 & 21,100 & 20,200 & 19,300 & 1.42 & 18 \\
\hline Q & SKSK-AQAAQAWAQA-KSKSKS & 1,500 & 19,400 & 20,000 & 19,200 & 1.11 & 19 \\
\hline Y & SKSK-AYAAYAWAYA-KSKSKS & 1,000 & 18,300 & 19,000 & 17,500 & 0.69 & 18 \\
\hline$T$ & SKSK-ATAATAWATA-KSKSKS & 2,300 & 16,600 & 18,700 & 16,600 & 0.83 & 18 \\
\hline $\mathrm{S}$ & SKSK-ASAASAWASA-KSKSKS & 3,000 & 16,200 & 17,900 & 16,000 & 0.77 & 19 \\
\hline $\mathrm{N}$ & SKSK-ANAANAWANA-KSKSKS & 1,900 & 13,800 & 16,000 & 13,300 & 0.67 & 19 \\
\hline G & SKSK-AGAAGAWAGA-KSKSKS & 500 & 12,100 & 15,500 & 12,100 & 0.57 & 17 \\
\hline$P$ & SKSK-APAAPAWAPA-KSKSKS & 300 & 7,400 & 3,100 & 2,700 & 0.57 & 18 \\
\hline
\end{tabular}

1 See Methods section for preparation and characterization of peptides. Single letter codes of amino acids are used.

${ }^{2}$ Mean residue ellipticity (deg cm $\left.\mathrm{dmol}^{-1}\right)$ of the peptides at $222 \mathrm{~nm}\left(5^{\circ} \mathrm{C}\right.$ in aq. buffer, $25^{\circ} \mathrm{C}$ in SDS, LPG, and DMPG). Values reported are based on triplicate measurements; estimated deviation, $\pm 1 \%$. Peptide concentration was $30 \mathrm{mM}$ for each peptide, determined by amino acid analysis ${ }^{13}$. Aq.: $10 \mathrm{mM}$ sodium chloride, $10 \mathrm{mM}$ phosphate, pH 7.0. SDS: $10 \mathrm{mM}$ sodium dodecylsulfate micelles in Aq., pH 7.0. LPG: 5 mM egg lysophosphatidylglycerol micelles in $10 \mathrm{mM}$ Tris-HCl, pH 7.0. DMPG: 3 mM unilamellar dimyristoylphosphatidylglycerol vesicles in $10 \mathrm{mM}$ Tris- $\mathrm{HCl}, \mathrm{pH}$ 7.0. See Methods for further details of sample preparation and CD measurements.

${ }^{3} \mathrm{P}_{\alpha}=$ Helical propensity parameter of amino acid $(X)$ as given on the Chou-Fasman scale ${ }^{5}$.

${ }^{4} \lambda_{\max }^{\alpha}=$ wavelength at which Trp fluorescence emission is maximal. For all peptides, $\lambda$ (aq.) $=355 \pm \mid \mathrm{nm}$. Blue shift for Trp emission, $D \lambda_{\max }=\operatorname{Trp} \lambda_{\max }$ (aq.) - Trp $\lambda_{\max }$ (SDS). Excitation: $280 \mathrm{~nm}$. Similar $\mathrm{D} \lambda_{\max }$ values were obtained for peptides in LPG micelles and DMPG vesicles (not shown). 